\title{
A la espera: la «drôle de guerre» en Un balcon en forêt
}

\author{
Jorge IsUSI GARTEIZGOGEASCOA \\ University Studies Abroad Consortium (USAC) Madrid \\ Universidad Rey Juan Carlos \\ jorge.isusi@urjc.es
}

\section{RESUMEN}

Se propone a continuación un breve comentario a una de las novelas emblemáticas de Julien Gracq, a partir de la propia experiencia del autor durante la llamada «drôle de guerre». Se trata de dar cuenta de en qué medida transfiguró Gracq dicha experiencia al trasponerla a la ficción, así como de analizar algunos de los rasgos estructurales, temáticos y estilísticos más característicos de la obra.

Palabras clave: Julien Gracq, drôle de guerre, espera, Un balcon en forêt.

\section{Awaiting: the drôle de guerre» in Un balcon en forêt}

\begin{abstract}
In the lines that follow I pose a commentary on one of Julien Gracq's best known novels, based on the author's own experience during the so-called «drôle de guerre». I aim to account for the way in wich Gracq transfigured that experience of his into a work of fiction. I also examine some of the structural, thematic and stylistic features of the novel.
\end{abstract}

KEYwORDS: Julien Gracq, drôle de guerre, espera, Un balcon en forêt.

\section{Apunte biográfico}

El 10 de mayo de 1940, el teniente de infantería del ejército francés Louis Poirier, profesor de Geografía e Historia en la vida civil que hace poco más de un año ha publicado una novela bajo el nombre de Julien Gracq ${ }^{1}$, se halla destacado junto a su unidad en la frontera con Bélgica. Ese día, de madrugada, lo despierta el ataque de aviones ale-

1 Au château d'Argol apareció en enero de 1939. 
manes: el ejército alemán invade Bélgica, los Países Bajos y Luxemburgo. Termina por fin de estallar la guerra, se pone así fin a nueve meses -recordemos que Francia y el Reino Unido habían declarado la guerra a Alemania el 3 de septiembre de 1939- de movilización a la espera de los acontecimientos, a ese período de la historia contemporánea de Francia que se ha dado en llamar la «drôle de guerre», la «guerra de broma».

Seguirán a esa jornada nuevos ataques, un traslado a Dunkerke a los pocos días, expediciones desde allí de avance y de repliegue, sin pies ni cabeza, por territorio belga y holandés y, finalmente, tras las capitulaciones de los ejércitos de estos dos países (los días 15 y 28 de mayo, respectivamente), la rendición de Poirier y sus hombres, a quienes se hace prisioneros en la tarde del 2 de junio. Al poco, se traslada a Poirier-Gracq a un campo de Silesia, donde permanecerá preso hasta febrero del año siguiente. Una enfermedad pulmonar provocará su liberación y su regreso a Francia: allí, a comienzos del mes de marzo, se reintegrará a la vida civil.

De parte de aquella experiencia en la guerra fue Gracq dejando testimonio directo a lo largo de los años en más de una entrevista, así como en diferentes textos autobiográficos incluidos en Lettrines y en los Carnets du grand chemin. (A estas referencias debemos añadir hoy un breve diario de guerra y una narración en tercera persona de los que solo a la muerte del autor, en 2007, se tuvo noticia y que se han publicado póstumamente en un mismo volumen con el título de Manuscrits de guerre). Partiendo de estas y otras referencias, se tratará de dar cuenta en este artículo de hasta qué punto y de qué manera está la experiencia de Gracq durante la «drôle de guerre» presente también en su obra novelística, en concreto, en Un balcon en forêt.

\section{Génesis y escritura de Un balcon en forêt}

Un balcon en forêt se publicó en 1958, siete años después de Le Rivage des Syrtes, la última novela de Gracq hasta la fecha. Hubo entonces, entre la crítica, quien acusó -la elección del verbo no es gratuita- a Gracq de haber escrito una novela realista, quien negó tal acusación, quien alabó la novela, quien le presentó reservas. Hoy pocos dudan de que se trata de una de las obras mayores de su autor.

Según la cronología que figura en la edición de las obras de Gracq en la Pléiade, edición a cargo de Bernhild Boie, experta en el autor, este comenzó la redacción de la novela en la primavera de 1956 (Gracq 1995: LXXVII). Tras la publicación y el éxito de crítica, no sin cierto escándalo por razones no estrictamente literarias, de Le Rivage des Syrtes, Gracq había empezado a escribir una novela vinculada, al menos formalmente, con aquélla, cuya redacción iba a interrumpir por esas fechas para siempre. (De aquella novela incompleta, extraería Gracq después un fragmento, que publicó en 1970 como una de las tres nouvelles independientes de La pres$q u$ 'ile, con el título de «La Route». Por lo demás, dicho texto se ha reintegrado recientemente a su lugar original en una edición póstuma de la citada obra inacabada con el título de Les terres du couchant (Gracq 2012). Entre los motivos que lo estimularon a tomar aquella decisión y ponerse a escribir lo que finalmente sería $U n$ balcon en fôret, el mismo Gracq solía citar el mero hecho de haber deseado desde hacía tiempo escribir algo acerca de la «drôle de guerre». En una entrevista de 1970 
(con Jean-Louis de Rambures), habla precisamente de ello y, para explicar su interés en el asunto, se refiere a la «drôle de guerre» como «una especie de temporada baja del alma de lo más particular» (Gracq 2002: 11)2. En la misma entrevista habla también de una larga excursión a pie por las Ardenas como de un hecho que lo marcó -sabemos que dicha excursión le serviría después para localizar los acontecimientos de la narración-, así como de la lectura de ciertas líneas de Los comunistas de Aragon por las que se enteró de la existencia, durante la guerra, de las llamadas «casas fuertes» de las Ardenas. El poder simbólico de esa imagen habría sido, según él, lo que puso definitivamente en marcha la composición del libro.

El hallazgo, a la muerte del autor, de dos cuadernos que contienen sendos manuscritos directamente relacionados con su experiencia en la guerra, han venido a ampliar y quién sabe si no a modificar las ideas que acerca de la relación entre la vida y la obra de Gracq se tenían hasta hace poco. Se trata, por una parte, de un diario de guerra escrito bajo la rúbrica Louis Poirier / Recuerdos de guerra que cubre los días que van del 10 de mayo de 1940 al 2 de junio; y por otra, de una narración sin título de 66 páginas de extensión, redactada en tercera persona, que tiene por protagonista al teniente G. y que cubre únicamente dos de esos días: el 23 y el 24 de mayo. Son los dos textos aludidos al principio del presente artículo, publicados en 2011 con el título de Manuscrits de guerre.

Las diferencias entre lo que se reproduce en estos dos textos y la historia narrada en Un balcon en forêt son notables: por un lado ambos se refieren a los últimos días de la «drôle de guerre», mientras que el Balcon cubre el curso de esta, a su manera, claro está, en su totalidad; por otra parte, ya era cosa sabida que la localización de los hechos narrados en el Balcon no coincide con los lugares en los que se desarrolló la experiencia guerrera de Gracq. El catálogo completo de las diferencias sería, ciertamente, prolijo. Ahora bien, cabe hacer notar por otra parte ciertas coincidencias, coincidencias que nos permitirían aventurar que al menos un primer germen literario de Un balcon en forêt podría retrotraerse hasta el mismo año de 1940 en que se redactaron los textos autobiográficos de los que venimos hablando. Así, en el diario, además de una atmósfera en muchas ocasiones parecida a la del último tramo del Balcon, encontramos, por ejemplo, ciertas vivencias del autor que en la novela pasará a protagonizar el aspirante Grange, como es el caso de la negativa de ambos en un momento dado a replegarse si no reciben una orden escrita, y aun algún símil que el narrador parece haber directamente «trasplantado» de un texto a otro: «Y después venía la ráfaga breve de la ametralladora del cuerpo de cazadores, semejante al crepitar de un bombo de lotería» (Gracq 2006: 148), dice la versión castellana del Balcon en la página 148, repitiendo prácticamente palabra por palabra la frase que cierra la entrada correspondiente al 10 de mayo del diario (Gracq 2011: 37). Por su parte, en la narración del segundo cuaderno se cuenta un episodio que tiene al teniente G. -elocuente tripleta, por cierto, la que forma esta inicial junto a las de Grange y Gracq- por protagonista junto con algunos de sus hombres. En él estos se hacen con el ominoso cargamento de un vagón de mercancías

\footnotetext{
2 La traducción es mía.
} 
que resulta no ser más que un conjunto de cartillas militares. Esas cartillas, si bien pasadas de nuevo por la imaginación y traspuestas bajo circunstancias distintas, tendrán también a su manera acomodo en el tramo final de la novela.

Gracq escribió la mayor parte del Balcon aprovechando, como era costumbre en él, períodos de vacaciones, concretamente los correspondientes a 1956 y 1957 . No deja de ser llamativo este hecho, el hecho de que escribiera durante las vacaciones, quiero decir, si tenemos en cuenta el «estado vacacional» en que se hallan los personajes de casi todas sus narraciones, de los cuales los de Un balcon en forêt no son una excepción. A ese estado de ociosidad se refirió el mismo Gracq, no solo en la «ficha descriptiva» de los personajes de sus novelas consignada en Lettrines, sino en distintas entrevistas a lo largo de los años.

Están - dirá de sus personajes a Jean Roudaut- siempre de vacaciones, o bien en la guerra, que es una clase particular de vacaciones, sujeta, a decir verdad, a tensiones brutales, pero donde la despreocupación, por lo demás, es por momentos total. (Gracq 2002: 68)

A ello volveremos más adelante en relación con nuestra obra.

\section{Estructura, voz narrativa, trama, espacio}

En contraste con el modo en que decidió organizar la narración en su novela anterior, Le Rivage des Syrtes, Gracq prescindió para la redacción de Un balcon en forêt de la división en capítulos. La narración se distribuye esta vez en veintiuna secciones de extensión variable separadas tipográficamente por un espacio en blanco. (La versión castellana, al menos en la edición publicada por Debolsillo en 2006, consta únicamente de veinte: las secciones $11^{\mathrm{a}}$ y $12^{\mathrm{a}}$ forman una sola por razones que ignoro. No es el único «pero» que puede oponerse a esta versión -más adelante se mencionará algún otro-, excelente, por lo demás, en casi todo).

Basta leer la primera frase de la novela («Una vez que su tren hubo atravesado los suburbios y humaredas de Charleville, etc.») -en rigor habría sido suficiente con leer las cuatro primeras palabras- para advertir que la historia que se despliega ante nosotros está narrada en tercera persona. En un primer momento, podría sorprender el hecho de que Gracq reserve precisamente la tercera persona para la que suele considerarse su novela más «autobiográfica». (Por otra parte, no olvidemos que también se empleaba la tercera persona en la narración de 1940, más «autobiográfica» todavía si cabe). Pareciera, sin embargo, que el distanciamiento connatural a la tercera persona fuese condición necesaria para asegurar el vuelo de la imaginación a partir de la experiencia de lo real -algo así como una manera, son palabras de Bernhild Boie, de «mantener gramaticalmente a distancia una ficción personal» (Gracq 1995: 1294)-, en un movimiento hasta cierto punto inverso al de la composición de Le Rivage des Syrtes, novela en la cual recordemos que es Aldo, el héroe, quien cuenta en primera persona su peripecia. Se deduciría que para evocar un mundo más o menos cercano, que parte de la propia experiencia, al autor le fuera necesario tomar 
distancia de él, mientras que para evocar otro tipo de mundo, lejano por puramente imaginario -la geografía y la historia, legendarias, de Le Rivage des Syrtes- le fuera, por el contrario, preciso «habitarlo» narrativamente en primera persona.

La trama de la novela es, como acostumbran serlo todas las de Gracq, sencilla. Un joven oficial del ejército francés, el aspirante Grange, llega una tarde de octubre de 1939 en tren, tras bordear el río Mosa, al puesto de mando de Moriarmé, situado en las Ardenas. Allí se lo destina al fortín de las Hautes Falizes, una casa fuerte en un alto del bosque, construida en hormigón y diseñada para impedir el acceso de blindados enemigos por las Ardenas Belgas, en la que vivirá junto a un cabo (Olivon) y dos soldados (Hervouët y Gourcouff) a sus órdenes los meses de la «drôle de guerre» (expresión, dicho sea de paso, que no se emplea como tal en ningún momento en la novela), con la excepción del tiempo que pasará de permiso en París, primero, y en la Turena, después, al final del invierno. Por cierto que también a Gracq se le concedió, en la vida real, un permiso antes de reincorporarse a su batallón, instalado ya en la frontera belga, a principios de abril (Gracq 1989: LXXI). Durante su estancia en el «Techo», como se conoce también a las Hautes Falizes, Grange permanecerá bajo las órdenes del capitán Varin, con quien mantendrá diferentes encuentros que a su modo callado van preludiando el desastre. En sentido parecido actuará la ominosa visita de un teniente de caballería que, antes de que llegue el invierno, le recomienda «cambiar de aires». En medio de todo ello se produce el encuentro con Mona, una joven viuda que está pasando una temporada en las Falizes junto a su criada; con ella vive Grange un idilio que ocupará un lugar central en la novela. Cuando por fin, la madrugada del 9 al 10 de mayo, llega el ataque alemán, los acontecimientos se precipitan: comienzan las explosiones, las retiradas, los cortes de comunicación con Moriarmé, el caos, en una palabra. A pesar de todo, Grange permanece junto a sus hombres en su puesto. Desde el blocao de la casa fuerte alcanzan una camioneta alemana (portadora de las cartillas militares a las que se ha hecho alusión antes) y al poco son alcanzados por un obús. En el ataque mueren Olivon y Hervoüet; Grange, herido de gravedad en varias partes del cuerpo, sale del blocao y huye con Gourcuff hacia el Mosa. En un momento dado, se separa de Gourcuff y se dirige a la aldea de las Falizes, evacuada desde hacía varios días. Consigue llegar a la casa de Mona (en una de sus últimas excursiones nocturnas se había guardado la llave de la casa en el bolsillo), se tumba en su cama y, finalmente, se duerme. Quizá no esté de más recordar que, como señala André Peyrone, tanto la primera sección como la última terminan de la misma manera, es decir, con el héroe, Grange, dormido (Peyrone 2007: 116); ignoramos si para siempre en el segundo caso: el punto final se impone para dejarnos en la incertidumbre.

Permítaseme un inciso, ya que hablamos del héroe, sobre un hecho que puede llegar a desconcertar al lector de la versión castellana de la novela. A la hora de reproducir en español el rango de «aspirant», los traductores ${ }^{3}$ optaron por el título español de «alférez», el cual, efectivamente, vendría a corresponder con aquél, si no en la denominación, al menos sí en cuanto a las posiciones que uno y otro rango ocu-

3 Jacqueline y Rafael Conte. 
pan en los respectivos escalafones. Parecería una decisión acertada si no fuera porque, a las pocas páginas de empezada la novela, advertimos que los subordinados de Grange en la casa fuerte y, más adelante, algún otro personaje, se dirigen a él empleando el vocativo «mi teniente», lo cual le hace preguntarse a uno si el ascenso físico desde Moriarmé hasta las Hautes Falizes no habrá supuesto un ascenso paralelo del personaje en el escalafón del ejército. No hay tal cosa: al parecer, en el ejército francés, todo aspirante recibe de sus subordinados el tratamiento de teniente.

Pero volvamos al escenario -la metáfora trasciende esta vez el lugar común- en que se desarrolla la acción. Se trata, por lo pronto, de un lugar fronterizo. Los personajes de Gracq tienden a ser, de forma recurrente, habitantes de la frontera: ya sea de los legendarios confines de Orsenna, más allá de los cuales se encuentra la indefinida amenaza del Farghestán, en Le Rivage des Syrtes; de las orillas de la costa bretona, en Un beau ténébreux; de la línea del río Mosa en las Ardenas, en la novela que ahora nos ocupa.

Para explicar la elección de este último espacio, del que Gracq apenas modificó el nombre de ciertos lugares (Monthermé pasará a ser Moriarmé en la ficción, los Haut-Buttés se transformarán en las Hautes Falizes, etc.), él mismo recurrió alguna vez al concepto de «paisaje-historia» (Gracq 2002: 31). Las Ardenas, no solo por sus características naturales, sino por haber sido el escenario de sucesivos desastres bélicos desde el siglo XIX, se prestaban como ningún otro lugar al tipo de historia que Gracq se propuso escribir y que finalmente escribió. Un lugar así fácilmente podía elevarse, en la imaginación de Gracq, de lo geográfico a lo poético y convertirse, como por momentos se sugiere en la novela, en otra cosa: en escenario de una representación o simulacro (la misma presencia de la palabra balcon en el título original -circunstancia que, dicho sea de paso, en la versión castellana se pierde- es elocuente en este sentido), en isla, y aun en bosque de cuento de hadas.

\section{A la espera. Tiempo histórico-tiempo narrativo}

Gracq, en entrevista con Jean Roudaut: «La historia, durante la «drôle de guerre», se transformó por un momento en espera pura, yo me sentía en comunicación imaginativa con ella» (Gracq 2002: 56) ${ }^{4}$.

Es sabido que la espera es un tema recurrente en la obra de Gracq. Es más, uno se vería tentado de decir que es el gran tema de Gracq. Y es que sus historias se centran y se demoran, fundamentalmente, en el tiempo en que sus personajes se mantienen a la espera de un acontecimiento. En el caso que nos ocupa, recordemos que a Grange y a sus hombres se les ha encomendado ejercer el papel de centinelas, cometido al que la espera es consustancial. En ese estado de suspenso -esa «clase particular de vacaciones», de las que hablaba Gracq- permanecerán durante la mayor parte de la novela.

\footnotetext{
4 La traducción es mía.
} 
Ya se ha sugerido que en Un balcon en forêt, Gracq hace coincidir el tiempo histórico de la «drôle de guerre» con el tiempo en el que se desarrolla la acción. Aunque esta se narra de forma lineal, mediante elipsis y referencias más o menos precisas al tiempo histórico («una tarde de octubre», «a finales de diciembre», «hacia el final del invierno», «En la noche del 9 al 10 de mayo», etc.), su desarrollo está sujeto a ciertas peculiaridades. Suzanne Allaire divide la narración en tres tiempos: el del principio de la novela, que nos sitúa propiamente en el contexto de la guerra, el tiempo que dura el idilio entre Grange y Mona, que Allaire, con buen criterio, concibe como una especie de paréntesis, y la vuelta al mundo dramático de la guerra a partir de la ofensiva alemana (Allaire 2007: 29-30). Si tomamos esta división como referencia, al primer tiempo corresponderían las cinco primeras secciones, al segundo, las secciones que van de la $6^{\mathrm{a}}$ a la $11^{\mathrm{a}}$, ambas incluidas, y al último, las pertenecientes al tramo final de la novela (secciones $12^{\mathrm{a}}-21^{\mathrm{a}}$ ). De este modo, la parte que ocuparía una mayor extensión sería precisamente la segunda, la que de algún modo representa un paréntesis respecto del progreso del tiempo histórico. Esta división no debe hacernos pensar que la novela esté concebida a la manera de compartimentos estancos: la narración, no obstante lo apuntado, fluye desde el principio hasta el final sin sobresaltos. Desde la primera frase de la novela, en la que se nos dice que una vez atravesado el tren de Grange los suburbios de Charleville a este «le pareció que la fealdad del mundo se disipaba», somos testigos de un viaje que en el fondo lo va a ser a un tiempo mágico, en suspenso, que trasciende las coordenadas históricas, y que tal vez alcanza su punto culminante con la caída, en la sección $9^{\text {a }}$, de las primeras nieves en el Techo. En pleno idilio con Mona, Grange siente «su vida tibia y latiente, libre de sus amarras, aislada de su pasado y su porvenir como los profundos saltos que separan las páginas de un libro.» Entramos en un tiempo estático - no en vano el uso del imperfecto es predominante a lo largo de estas páginas-, y extático, pues la felicidad de Grange, reflejada en una especie de comunicación íntima con su circunstancia, es una felicidad plena, sin porqué (nótese que cuando el capitán Varin, ante la sospecha de un inminente ataque alemán, le ofrece a Grange un traslado al regimiento, este lo rechaza, a sabiendas del peligro que corre, con el único motivo de encontrarse «a gusto» allí) $)^{5}$. No faltan, en medio de dicha plenitud signos de la catástrofe que está por llegar (ahí están, entre otros, las citadas conversaciones con el capitán Varin y la visita del teniente de caballería, el vuelo de un avión alemán de reconocimiento, así como, en un orden diferente, las ansiedades nocturnas de Mona o el sueño que el mismo Grange tiene la víspera de su regreso a la casa fuerte tras el tiempo de permiso), signos que contribuyen precisamente a dotar de continuidad a la narración. Ya a la vuelta de su permiso, el Techo no le parece a Grange «el mismo». «¡Esto se mueve!» exclamará Olivon. De algún modo se ha roto el encanto, la Historia, que hasta ahora ha permanecido hasta cierto punto ausente, hará por fin acto de presencia: será hora de despedirse de Mona, en breve se despejará «la bruma de la falsa guerra»: llegará, en suma, lo que ya sabemos.

5 En cierto modo, cabría asimilar este tiempo de felicidad al tiempo consuntivo del que -por oposición al tiempo adquisitivo- da cuenta Rafael Sánchez Ferlosio en, por ejemplo, «Juegos y deportes» (Sánchez Ferlosio 2000: 481-482). 


\section{Coda}

En una entrada de Lettrines 2, Gracq escribe lo siguiente: «Los recuerdos de la guerra de 1940, que durante dieciocho años había conservado tan vivos y tan precisos, una vez escribí Un balcon en forêt se perdieron en la confusión y en la oscuridad» (Gracq 2008: 113). De este particular «empobrecimiento» del escritor de novelas habló en más de una ocasión. Algo muy similar, por cierto, a lo que Nabokov, otro grande del siglo XX, dejó escrito respecto de su propia obra en Speak, Memory (Nabokov 1989: 95). Solo nos cabe, en fin, agradecer al cielo que a su debido tiempo ninguno de los dos dudara en sacrificar sus recuerdos personales a la escritura, pues la desmemoria biográfica que asumieron al hacerlo es hoy parte de nuestra más preciada memoria literaria.

\section{Referencias bibliográficas}

ALLAIRE, Suzanne (2007): «Un balcon en forêt. Les pouvoirs de l'écriture: liberté grande», en Lectures de Julien Gracq: Un balcon en forêt, La Presqu'île, Frank Wagner (ed.), pp. 25-42, Presses Universitaires de Rennes, Rennes.

GRACQ, Julien (1989): Euvres complètes, vol.I, Gallimard, Bibliothèque de la Pléiade, París.

GraCQ, Julien (1995): Euvres complètes, vol.II, Gallimard, Bibliothèque de la Pléiade, Paris.

- (2002): Entretiens, José Corti, Paris.

- (2006): Los ojos del bosque, Debolsillo, Barcelona.

- (2008): Lettrines 2, José Corti, Paris.

- (2011): Manuscrits de guerre, José Corti, Paris.

NaвOKOV, Vladimir (1989): Speak Memory. An Autobiography Revisited, Vintage International, New York.

PEYRONE, André (2007): Un balcón en forêt de Julien Gracq: les guetteurs de l'apocalypse, Lettres modernes Minard, Caen.

SÁNCHEZ FERLOSIO, Rafael (2000): El alma y la vergüenza, Destino, Barcelona. 